ELECTRIC COHEATING AS A MEANS TO TEST DUCT EFFICIENCY: A REVIEW AND ANALYSIS OF THE LITERATURE

RECEIVED

NOV 2 y 1995

J. W. ANDREWS

OSTI
Energy Efficiency

and Conservation Division

DEPARTMENT OF APPLIED SCIENCE
AUGUST 1995

Prepared for:

Office of Building Technologies

Building Equipment Division

U.S. Department of Energy

Washington, DC 20585
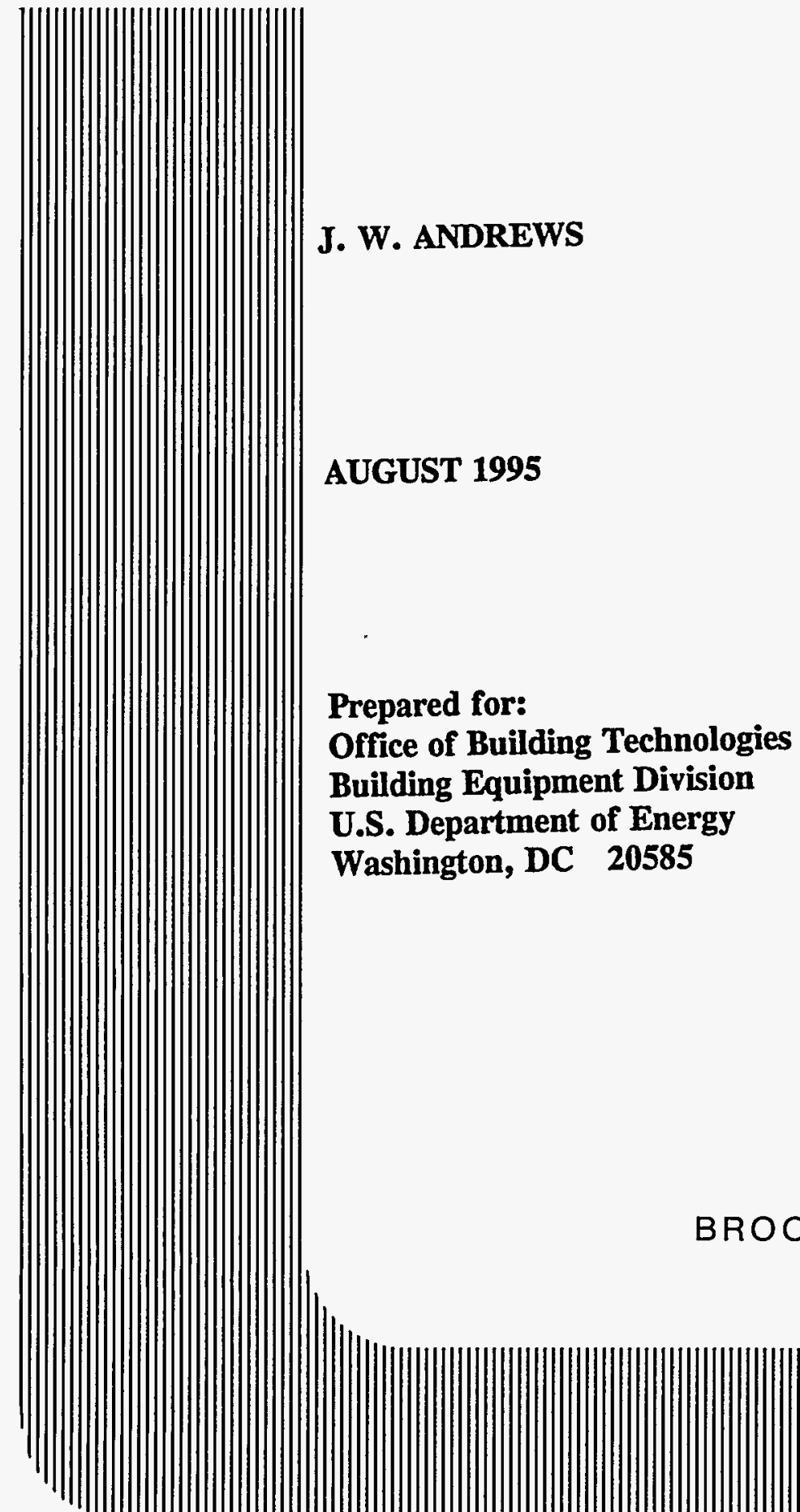
UPTON, LONG ISLAND, NEW YORK 11973
BROOKHAVEN NATIONAL LABORATORY

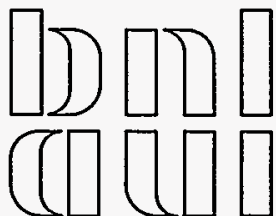

DISTRIBUTION OF TH:S DOCUMENT IS UNLMATED 


\title{
ELECTRIC COHEATING \\ AS A MEANS TO TEST DUCT EFFICIENCY: \\ A REVIEW AND ANALYSIS OF THE LITERATURE
}

\author{
J. W. Andrews
}

August, 1995

\begin{abstract}
Prepared for:
Building Equipment Division

Office of Building Technologies

U.S. Department of Energy

Under Contract No. DE-AC02-76CH00016
\end{abstract}

Brookhaven National Laboratory

Upton, New York 11973

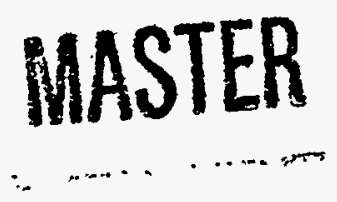




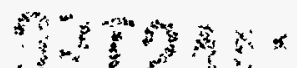

tैen 


\section{Abstract}

Recent published literature on electric coheating was reviewed in order to assess its suitability for use in a method of test for the efficiency of residential duct systems. Electric coheating is the research use of electric heaters within the heated space to assess the thermal integrity of the building envelope. Information was sought in two primary areas: (1) experimental methodology and (2) accuracy of the coheating method. A variety of experimental variations was found, and the method was judged, on the basis of published data, to be capable of sufficient accuracy for use in duct testing.

\section{DISCLAIMER}

This report was prepared as an account of work sponsored by an agency of the United States Government. Neither the United States Government nor any agency thereof, nor any of their employees, makes any warranty, express or implied, or assumes any legal liability or responsibility for the accuracy, completeness, or usefulness of any information, apparatus, product, or process disclosed, or represents that its use would not infringe privately owned rights. Reference herein to any specific commercial product, process, or service by trade name, trademark, manufacturer, or otherwise does not necessarily constitute or imply its endorsement, recommendation, or favoring by the United States Government or any agency thereof. The views and opinions of authors expressed herein do not necessarily state or reflect those of the United States Government or any agency thereof. 


\section{Table of Contents}

Abstract . . . . . . . . . . . . . . . . . . . . i

Introduction . . . . . . . . . . . . . . . . . . . 1

Electric Coheating Methods . . . . . . . . . . . . 3

The Dawn of Coheating . . . . . . . . . . . . 4

A Simple Approach to Coheating. . . . . . . . . 、 5

Measured Performance Rating . . . . . . . . . 6

The PSTAR/STEM Approach . . . . . . . . . . . 7

Coheating for Duct Efficiency Testing . . . . . . 9

Accuracy of Coheating. . . . . . . . . . . . . . . . 11

Repeatability of Results. . . . . . . . . . . 11

Systematic Error. . . . . . . . . . . . . . 15

Systematic Error Quantified in Duct
Efficiency Testing . . . . . . . . . . 17

Consistency of Coheating Results with
Physical Constraints.............. 21

What About Cooling?. . . . . . . . . . . . . . . . . 22

Conclusions. . . . . . . . . . . . . . . . . . . 23

Recommendations. . . . . . . . . . . . . . . . 23

References . . . . . . . . . . . . . . . . . . . 24

\section{List of Tables}

1. Elements of the Coheating Protocol . . . . . . . . 3

2. Random Errors in Heating Load Coefficients . . . . . 15

3. Summary of Biases from Phase Lag of Heating Load with Respect to Temperature . . . . . . . . . 18 



\section{Introduction}

Recent published literature on electric coheating was reviewed in order to assess its suitability for use in a method of test for the efficiency of residential thermal distribution systems. Thermal distribution systems are the ductwork, piping, or other means used to transport heat or cooling effect from the building equipment that produces this thermal energy to the spaces in which it is used. Electric coheating is the use of electric heaters within the heated space to assess the thermal integrity of the building envelope. The thermal energy delivered per unit time by the heaters, divided by the average indoor-outdoor temperature difference, yields a parameter which we call the Heating Load Coefficient (HLC). (Other names for this quantity, which has dimensions of energy over the product of temperature and time, include building load coefficient and thermal loss coefficient.) We will reserve the term "UA value," which has the same units, to denote the "true" heat-loss coefficient under ideal steady-state conditions. Naturally, one would like the HLC to be as close as possible to the UA value; however, as will be seen in the discussion of systematic error, one can tolerate situations where this is not strictly true, as long as certain conditions are met.

Three quite different methods have been used to quantify the effect of thermal distribution on overall heating (or cooling) system efficiency. By far the most common method has been to measure the individual factors that contribute to energy losses in ductwork, such as conductive losses, air leakage, and fan-induced infiltration. [Caffey 1979, Cummings et al. 1989, Gammage et al. 1984, Matthews et aI. 1990, Modera 1989, Parker 1989, Palmiter and Bond 1992, Proctor et al. 1992, Robison and Lambert 1989] A second method has been to compare, on a seasonal basis, the heating energy consumption of two large samples of homes, where one sample is heated with electric furnaces and duct systems while the second sample uses electric baseboard heating. [Lambert and Robison 1989]

The third method uses electric coheating. The coheating system measures the "bare" heating load of a house without equipment or distribution losses, which is then compared with the energy used by the ducted heating system. One group has used coheating to determine thermal distribution efficiency in homes with electric furnaces and heat pumps. [Palmiter and Francisco 1994, olson et al. 1993] More recently, the author of this report, together with coworkers, used coheating to measure thermal distribution efficiency in two houses. [Andrews et al. 1995a, 1995b]

All of these methods have led to the same general conclusion, that duct systems typically lose 25 to 40 percent of the energy output from the space-conditioning equipment. In response to this information, the American Society of Heating, Refrigerating, and Air-Conditioning Engineers (ASHRAE) has established standards Project Committee (SPC) 152P, Standard Method of Test for 
Determining the steady-state and Seasonal Efficiencies of Residential Thermal Distribution Systems.

Electric coheating has been proposed as a research tool to be used in standard 152. Coheating would be used to obtain system efficiency as the dimensionless ratio of the HLC measured via coheating to a similar parameter that includes the thermal performance of the building's own heating system. This second parameter is called the Heating system Coefficient (HSC). System efficiency $\eta_{\text {system }}$ is then defined as the ratio of HLC to HSC. For heating systems that use fuel-fired furnaces, this ratio will always be less than 1.0, the extent to which it falls short of unity accounting for all the thermal losses in both the equipment and the distribution system, including any interactive effects on the heating load. (For a system served by a heat pump, $\eta_{\text {system }}$ can exceed 1.0.) System efficiency is important not only in its own right, but also because it serves as the starting point for determining the thermal distribution efficiency. Distribution efficiency is calculated by dividing system efficiency by the efficiency of the heating or cooling equipment and then correcting for interaction between equipment and distribution system efficiency. Further information on how this test method is being conceptualized is given in Modera et al. 1992 and, at a more basic level, in Andrews 1994.

The test protocol currently envisaged for ASHRAE standard 152 utilizes both heating system and coheating tests. on each night of a two-night evaluation. Nighttime testing is proposed to minimize the effect of solar gain and to maximize indoor-outdoor temperature difference. Doing both kinds of tests on both nights was selected over the alternative of doing system testing on one night and coheating on the other in order to minimize bias that might result from large changes in weather conditions between the two nights. This way of doing the tests has been called the "flip-flop protocol." A summary of the coheating test protocol currently envisaged for ASHRAE standard 152, incorporating the flip-flop protocol, is given in Table 1 .

A comprehensive review and analysis of the thermal distribution literature was performed three years ago. [Andrews and Modera 1992] More recently, the need was seen for an updated search that would focus on coheating. This report discusses the information gained in the course of the new search and the conclusions we have derived from it. The two major goals of the search were:

- To establish the range of research applications in which coheating has been used; and

- To assess the accuracy of coheating, both in itself and as applied to thermal distribution testing. 
Table 1. Elements of the Coheating Protocol Proposed for ASHRAE Standard 152 .

\begin{tabular}{|c|c|}
\hline $\begin{array}{l}\text { Time } \\
\text { Frame }\end{array}$ & Test Descriptions \\
\hline Day 1 & $\begin{array}{l}\text { Cover south, east, and west windows with reflective } \\
\text { foil. Set up and check out instrumentation. }\end{array}$ \\
\hline $\begin{array}{l}\text { Night } 1 \\
6 \mathrm{p} \cdot \mathrm{m} .- \\
12 \mathrm{p} \cdot \mathrm{m}\end{array}$ & $\begin{array}{l}\text { Operate heating system and measure its energy use. } \\
\text { Compute first night's HSC, taking account of } \\
\text { indoor-outdoor temperature difference, test } \\
\text { duration, and internal energy gains. }\end{array}$ \\
\hline $12-1$ a.m. & Slack time for transients to die out. Coheaters on. \\
\hline $\begin{array}{l}\text { Night } 1 \\
1 \text { a.m.- } \\
7 \text { a.m. }\end{array}$ & $\begin{array}{l}\text { First coheat test. Compute first night's HLC. } \\
\text { Compute first night's system efficiency } \\
\eta_{\text {system }}=\text { HLC/HSC. }\end{array}$ \\
\hline Day 2 & $\begin{array}{l}\text { Optional tests, such as house and duct leakage. } \\
\text { Coheaters running during the day. }\end{array}$ \\
\hline $\begin{array}{l}\text { Night } 2 \\
6 \text { p.m. - } \\
12 \text { p.m. }\end{array}$ & Compute second night's HLC. \\
\hline $12-1$ a.m. & Slack time. Heating system on again. \\
\hline $\begin{array}{l}\text { Night } 2 \\
\text { I a.m.- } \\
7 \text { a.m. }\end{array}$ & $\begin{array}{l}\text { Second heating system test. Compute second night's } \\
\text { HSC, } \eta_{\text {syster. Average two system efficiencies to get }} \\
\text { a best value. }\end{array}$ \\
\hline
\end{tabular}

Electric Coheating Methods

In examining the variety of approaches to coheating that have been used in the field, we found the following two questions to be especially useful in organizing the information gained:

o How is the coheating system controlled?

- What other measurements and calculational procedures are used along with coheating?

It was found that the experimental methods and control systems ranged from the simple (coheaters controlled by individual thermostats) to the complex (computer-controlied systems coupled with a variety of additional measurements and simulations). The following papers are discussed, in rough order from the simplest approach to the most involved, with priority to the inventors of the method. 
The Dawn of Coheating. Sonderegger et al. (1979) published the first description of the coheating method. They used portable electric heaters controlled by a computer system. In their setup, the heaters were grouped into two clusters of four heaters each, with the heaters in each group cycled on for times proportional to the temperature difference between the setpoint and the room, i.e. the degree to which the room temperature has "drooped" below its setpoint. This is called proportional control.

The heaters were activated, and after a waiting period of 3 to 5 hours, the system was considered to have settled down enough to begin taking data. Tests were performed at night, to minimize the impact of solar gains. Power was measured using a pair of utilitytype watt-hour meters (one for each cluster of heaters). The house watt-hour meter was used as a backup.

Air infiltration was measured using the controlled-flow tracer gas method, in which a tracer gas is injected into the living space at several locations and the concentration in the return duct is measured.

In their discussion, the authors of this paper covered various procedures that they actually carried out:

- Using the coheaters to meet the entire heating load of the house, in order to measure the heating load coefficient.

- Inputting a specified amount of heat with the coheaters while allowing the furnace to run under control of its thermostat. Heating system efficiency was calculated as the electrical input of the coheaters divided by the reduction in fuel use caused by the addition of electric heat.

- Cycling the furnace in a preprogrammed way while using the coheaters to maintain the setpoint temperature. In this case the heating system efficiency was calculated as the reduction in coheat power divided by the fuel used by the furnace.

The authors felt that the second of these two ways of determining system efficiency is better because it gives more repeatable results.

- Using the coheaters to assess the thermal efficiency of a fireplace.

- Using coheaters to add a known cooling load, to assess airconditioning efficiency as the increase in cooling load divided by the increase in power consumption of the air conditioner.

Sonderegger et al. view electric co-heating as having several advantages and disadvantages. From the point of view of thermal 
distribution testing, the main advantage cited by the authors is "its ability to measure heating and cooling efficiencies in-situ, at realistic operating conditions." The main disadvantages seen by the authors are the need to do experiments at night, the need for a sophisticated control system, and the large electrical demand on the house wiring, which in some cases may not be able to deliver enough power to handle the number of heaters that are needed.

A Simple Approach to Coheating. On the spectrum from straightforward to complex, the paper that appears to be closest to the former is Duffy et al. (1989). This paper, reporting on work done for the National Association of Homebuilders Research Center, describes a building energy monitoring and analysis procedure that uses electric coheating as a fundamental means of obtaining the building load coefficient.

A major characteristic of the approach discussed in this paper is the idea that coheating tests are relatively simple to do. The authors state that "the coheating...tests can be performed with glass thermometers, portable heaters, clock timers, and the existing electric watt hour meter." If individual thermostats are used, they recommend a deadband of less than 2 F. They also say, however, that there is nothing wrong with using a computer control system. They say as well that the tests are relatively independent of outside weather conditions, though they do recommend doing the tests when the outdoor temperature is as low as possible to improve accuracy. Other recommendations include:

- Cover all windows on the east, south, and west sides of the house to minimize the impact of solar gains.

- Precondition the house for at least one day to stabilize thermal mass, unless thermal mass temperatures are monitored.

- Monitor both heater on-time and the house watt-hour meter to provide an independent check on total power consumption.

o. Measure windspeed at $10 \mathrm{~m}$ above the ground.

- For basement houses, it is recommended that a second coheating test be performed in which the basement temperature is maintained the same as that of the rest of the house. This enables the ground-coupling factor to be determined.

- A blower-door test should be performed. Infiltration should be measured directly using a tracer gas method, and this measurement used to adjust the long-term infiltration predicted using the blower-door test.

- They also recommend a cooldown test to determine the thermal time constant of the house. 
In general, the tone of this paper is that coheating, when augmented by other tests such as infiltration, blower door, and windspeed tests, is a not-too-difficult, repeatable means of obtaining overall heating system performance (which is called system efficiency in the SPC152P literature).

Another report on the same research program as the above [Spears 1985] described the use of electric coheating to measure the linear component of the response of a building's heating load to indoor-outdoor temperature differences, which is the same as the heating load coefficient defined in the Introduction. Spears recommended that coheating be done on three to five nights to average effects of wind, temperature differential, mass, humidity, and other factors.

What Spears calls the "delivered efficiency of the HVAC system" is measured in essentially the same way as system efficiency in the current SPC152P draft. Spears states that "the result is the actual in situ delivered efficiency of the HVAC system taking into account both the combustion efficiency of the furnace or boiler and the distribution losses."

Measured Performance Rating (MPR). In an effort to put electric coheating on a codified basis that would find acceptance in the housing industry, a methodology called Measured Performance Rating (MPR) was developed. [Saunders et al. 1994] MPR is intended as a relatively low-cost method to be used in assessing the effectiveness of weatherization procedures such as insulation and measures to reduce air infiltration. The MPR method uses four tests:

1. Coheat Test. This is recommended to take place for a minimum of six nighttime hours. A heating load coefficient, taken as a measure of the UA-value, is estimated as the average coheating power required (in $B t u / h$ or $W$ ) divided by the average indooroutdoor temperature difference.

2. Heating System Efficiency Test. In this test, the heating system is run for at least four complete on-off cycles. This is said to take typically three to four hours. The heating system efficiency (equivalent to $\eta_{\text {system }}$ in the SPC152P terminology) is calculated as the net heat added by the heating system divided by its fuel consumption. The net heat added is computed as the difference between the heating load and the internal gains, where the heating load is computed from the heating load coefficient (as found during coheating) and the indoor-outdoor temperature difference; and the internal gains (mainly electrical) are estimated from the electric power consumption.

3. Air Infiltration Test. Air infiltration rates during the tests are measured using $\mathrm{SF}_{6}$ tracer gas, either by collecting samples and sending them out for analysis or by doing on-line analysis with 
one's own gas chromatograph. The former method was said to cost $\$ 60$ to $\$ 120$ per test, while the latter required a capital investment of $\$ 7,000$ to $\$ 10,000$.

4. Blower Door Test. Measurement of the effective leakage area with a blower door, coupled with the LBL infiltration model, provides a way to estimate seasonal average air infiltration. A corrected-average heating load coefficient is then computed by subtracting the measured infiltration heat loss rate (using tracer gas) and then adding back the computed seasonal average infiltration heat loss rate (based on the blower-door test).

The MPR method as described here contains the germ of the approach contemplated for use in ASHRAE standard 152 for the direct measurement of system efficiency, with, however, significant differences in detail.

The PSTAR/STEM Approach. Subbarao (1988a) summarizes the philosophy and structure of a building performance analysis method developed at the Solar Energy Research Institute (now the National Renewable Energy Laboratory). It is called Primary and Secondary Terms Analysis and Renormalization (PSTAR). It is relevant to the present discussion because it relies on electric coheating for much of the input information to the analysis.

The starting point of this approach is the realization that buildings are complex, and that therefore accurate modeling of the thermal response of a building requires a complex model. However, measurements of building performance usually do not obtain anywhere near enough information to drive these complex models satisfactorily. Subbarao resolves this dilemma by using an approximation-correction procedure.

An initial set of building energy performance parameters is obtained by means of an energy audit conducted by an experienced professional. It is fully acknowledged that this audit will only give an approximate picture of the building. Certain short-term tests are then performed, on the basis of which the model is "renormalized" to give a more accurate picture of its actual performance.

The method is based on an energy balance involving the following generic terms:

- Internal energy gains

- An envelope heat loss coefficient or UA value, multiplied by the indoor-outdoor temperature difference.

- Thermal mass effects coupled to the indoor temperature.

- Thermal mass effects coupled to the outdoor temperature. 
- Solar heat gains.

- Heat loss caused by infiltration and ventilation.

- Heat supplied by the heating system.

o Heat flow to the ground or to the basement.

- Heat loss caused by sky temperature depression.

These terms are divided into three categories: those that are considered to be known (e.g. internal gains, heat supplied by the heating system); those that are not known but are of primary importance (e.g. envelope heat loss coefficient, thermal mass effects coupled to the indoor temperature, solar gains); and those that are of secondary importance. The treatment of these terms is as follows:

o The known terms are taken as measured.

- The secondary terms are accepted as modeled.

- For the primary terms, short-term tests are performed, on the basis of which the model is "renormalized" with respect to these primary terms.

A more lengthy report [Subbarao 1988b] provides detailed information on the theory behind PSTAR, emphasizing the impact on transfer functions and their evaluation. It demonstrates the flexibility of PSTAR and its ability to ferret out a variety of building characteristics, but its scope is very much beyond what is needed for the type of test that SPC152P is evolving into, which is much simpler.

Complementing the above theoretical approach is a specific protocol. Subbarao et al. (1988c) intended to elicit the renormalization factors for the three primary terms: steady-state thermal loss coefficient; thermal mass (internal); and solar gains. A nighttime coheating test is used to evaluate the envelope heat loss. A nighttime cooldown test is used to evaluate thermal mass impact. A daytime coheating test evaluates the effects of solar gains. This renormalization gives a much more accurate picture of the building's performance under any conditions. In particular, it then permits the renormalized model to accurately predict the seasonal energy use. The protocol described in this paper is called Short-Term Energy Monitoring (STEM). That is, STEM is a specific experimental protocol that makes use of the PSTAR theory to quantify building performance.

The relevance of all this to SPC152P is that in the discussions that led up to the current approach to the use of coheating, the assumption was made that thermal distribution 
efficiency is not a strong function of the thermal mass effects or of the solar gain, and so every effort was made to perform the tests under conditions where these effects are minimized. The only remaining primary term is then the envelope heat loss coefficient.

A later paper in this NREL series [Balcomb et al. 1993] describes the PSTAR-STEM process to a wider ASHRAE audience. It also describes the use of the building as a calorimeter to assess effects not included in the basic STEM procedure. Performance of a heating system (including ductwork) is the example given. That is, after the heating load coefficient has been determined using co-heating, the existing heating system is operated and its energy use compared with what the renormalized PSTAR model predicted would have been used with coheaters. The ratio of these two quantities is the system efficiency. Of five houses tested, four had system efficiencies below $40 \%$. Duct repairs were done on two of these houses, which showed great improvement on retesting.

With respect to SPC152P, which is taking a conceptually simpler approach to comparing the coheating with the system test, the full-blown PSTAR-STEM process might be useful as part of an effort to validate the SPC152 test method experimentally.

Coheating for Duct Efficiency Testing. As mentioned in the Introduction, the use of electric coheating as a way to obtain duct system efficiency was pioneered in the Pacific Northwest by the group led by Larry Palmiter. A comprehensive report [olson et al. 1993] discusses the results of electric coheating measurements in 24 Pacific Northwest homes. These homes had ducted electric heating systems (either electric furnaces or heat pumps). "Two of the homes had all the ductwork in the conditioned space; the rest had most of their ducts in unconditioned spaces.

The salient feature of the method used by this research group is that coheating and heating with the home furnace are alternated over relatively short intervals averaging 1.75 hours. Short intervals are intended to insure that the conditions under which the coheat and heating-system tests are carried out are as nearly the same as possible. Only the second half of each of these periods is used as data, to provide time for switchover transients to die down. The furnace period is programmed to cover an integral number of cycles, from fan-off to fan-off. The use of short intervals, however, requires that the furnace be controlled by the computer and not by the house thermostat, so that the number of cycles per hour can be held to between 6 and 8 and the furnace-on intervals will be similar to one another. Switchover between coheating and furnace heating is done automatically, so that the field crew does not have to be at the house all night. This also means, however, that registers are not sealed during the coheat period. 
The test protocol is summarized as follows:

- Initial information on the house; sketch of duct system; heating plant and thermostat information.

- Quick air flow measurement using electric resistance coil power and temperature rise across coil.

- Pressures across the air handler.

- Power draw of staged resistance coils, determined by turning the thermostat all the way up and measuring times and wattages for each stage.

- Register air flows using flow hood.

- Tracer gas infiltration measurement.

- Blower door test with registers open and sealed.

- Furnace and coheat test, alternating at 1.75 hour intervals.

System efficiency, calculated as the ratio of coheat energy to furnace electric energy input (per unit time in each case), averaged $71 \%$ for the 22 houses with ducts in unconditioned spaces. Since electric resistance coils provided the heat to the ducts, this was also the best estimate of distribution efficiency. For the two homes with ducts in conditioned spaces, system efficiency averaged 98\%. This was preliminary evidence that putting ductwork in the conditioned space is a good way to eliminate duct energy losses; - however, the authors called for additional tests on conditioned-space ductwork to confirm this. These tests also were relevant to the question of coheating's reliability as a test method (see below).

Tracer gas measurements showed an increase from 0.23 to 0.63 air changes per hour when the fan was turned on. Duct leakage area averaged $19 \%$ of total house leakage area. Both these values are consistent with other researchers' results. 


\section{Accuracy of Coheating}

Several papers provided insights into the accuracy of coheating as a test method, both in itself and as it is expected to be used in evaluating duct system efficiency. These fell into the following classes:

- Papers that provided evidence concerning the repeatability of coheating results.

- Papers that discussed possible sources of systematic error.

- Papers that provided direct evidence concerning the reasonableness of coheating-derived values of system efficiency.

Repeatability of Results. The earliest paper on coheating, Sonderegger et al. (1979), obtained a heating load coefficient in each of the variety of tests they did (see above). They expressed concern that the measured values of this coefficient varied considerably depending on whether they were testing the heating system, a fireplace, a cooling system, or measuring the infiltration load by pressurizing the house with a blower door. The values went from a low of $196 \mathrm{~W} / \mathrm{C}(370 \mathrm{Btu} / \mathrm{F}-\mathrm{h})$ to a high of $241 \mathrm{~W} / \mathrm{C}(450 \mathrm{Btu} / \mathrm{F}-\mathrm{hr})$. However, within one kind of test, the values were much more nearly constant. One possible reason for this might be that tests of a given kind were done close together in time, so that external weather-related effects might be more similar for tests of a given kind than for pairs of tests in general.

The first table in the referenced paper gives the following values for the building load coefficient found during four different tests, each aimed at a specific building subsystem. For each test, two values (called "initial period" and "final period") taken on the same night are recorded:

System Tested Building Load Coefficient (W/C)

$$
\text { Initial Period Final Period }
$$

$\begin{array}{lll}\text { Heating System } & 196 & 195 \\ \text { Fireplace } & 248 & 231 \\ \text { Cooling System } & 205 & 221 \\ \text { Infiltration Test } & 238 & 241\end{array}$

The average value of all the results is $222 \mathrm{~W} / \mathrm{C}$, with a standard deviation of $20 \mathrm{~W} / \mathrm{C}$, or about $9 \%$ of the mean value. If we take the unsigned differences between the initial and final period results in each case (i.e. $1,17,16$, and $3 \mathrm{~W} / \mathrm{C}$ ), and determine the standard deviation of these values, we obtain $7.3 \mathrm{~W} / \mathrm{C}$, which is only $3.3 \%$ of the original mean value. That is, the variability of the difference between two values obtained on the same night was 
slightly more than a third of the overall variability of the measured values. These results can be compared with those of Subbarao et al. discussed below.

Duffy et al. (1989), which was discussed briefly above, provided an engineering judgment concerning the relationship of coheat accuracy to outdoor temperature. Their

estimate was as follows:

Temperature Difference

$$
\begin{array}{ll}
10 & F \\
20 & F \\
30 & F \\
40 & F
\end{array}
$$

Expected Uncertainty

$$
\begin{array}{r}
10.0 \% \\
5.0 \% \\
3.3 \% \\
2.5 \%
\end{array}
$$

Jones et al. (1984) made two electric co-heating tests of a research house built in 1980 on Long Island (the Brookhaven House). The tests were done during the winter of 1980-81. The values for the heating load coefficient, obtained 20 days apart, were 335.7 and $344.6 \mathrm{Btu} / \mathrm{h}-\mathrm{F}$. These values differed by $2.6 \%$ of their mean, with a standard deviation $(\mathrm{N}=2)$ of $1.3 \%$ of the mean. The average indoor-outdoor temperature difference for these tests was $49 \mathrm{~F}$.

Duffy and Puri (1985) treat possible methods of extrapolating from short-term monitoring to long-term thermal performance. They predicate their analysis on the supposition that the short-term data will be taken using several measurements techniques, of which the first is electric coheating. Others include tracer-gas infiltration measurements, blower-door testing, and a cooldown test. It is suggested that only one or two nights of monitoring might be needed, if the building's interior temperature is stabilized during the tests.

In one approach to analyzing the short-term results, the authors found that "coefficient values derived from only 24 hours of data were extremely erratic. Coefficient values estimated on the basis of 96 hours ( 4 days) of data were reasonably close to those based on data for the entire month."

Subbarao et al. (1990) discuss the measurement of heating load coefficients using electric coheating. They state that "repeated measurements have been known to show considerable variation from night to night." The reasons given by these authors are: "storage effects due to variations in inside and outside temperatures, solar radiation, sky temperature depression, variable infiltration, and heat flow through the floor."

The main feature of the paper is their treatment of 13 coheating tests performed on a $95 \mathrm{~m}^{2}$ house on various nights over a five-month period January-May 1988. In each case a "raw" HLC was 
obtained from a two-hour period toward the end of each coheating period. The "raw" HLC's ranged from 121.1 to $206.7 \mathrm{~W} / \mathrm{C}$, with a mean value of $146.9 \pm 24.7 \mathrm{~W} / \mathrm{C}$. A check on the mean value using 13 HLC's read from the author's' Figure 1 gave a mean of 148.5 and a standard deviation of 24.5. On this basis we will assume (although the author doesn't say this explicitly) that his quoted error bars are in fact population standard deviations (calculated using $\mathrm{N}$ as the denominator) and not sample standard deviations (calculated using $\mathrm{N}-1$ ) or confidence intervals.

Two corrections were made, one to account for variations in infiltration and one to account for the fact that heat loss to the basement is driven by a lower temperature difference than heat loss to the outside. After these corrections were made, the average value shifted slightly, to $151.6 \mathrm{~W} / \mathrm{C}$, but the variability became much less, $7.8 \mathrm{~W} / \mathrm{C}$. Then four additional corrections were applied, for the following effects: storage effects of thermal mass coupled to the inside, storage effects of thermal mass coupled to the outside, solar gains, and night sky temperature depression. The magnitudes of these corrections were as follows (in W/C): instorage, $1.4 \pm 5.3$; out-storage, $4.7 \pm 2.3$; solar gains, $6.9 \pm 3.1$; and sky temperature correction, $-5.4 \pm 3.5$. After applying these corrections, a "corrected" HLC was obtained, whose average value for the 13 tests was $159.3 \pm 4.4 \mathrm{~W} / \mathrm{C}$.

Thus, the author obtained a standard deviation for all 13 HLC values equal to nearly $17 \%$ of the mean value, while after all corrections were made, this measure of variability dropped to less than $3 \%$ of the mean.

One might make the argument that Subbarao's corrections normalized the conditions under which the coheating tests were made, so that they became, in effect, about as similar as those obtaining in the successive measurements of sonderegger et al. discussed above.

NAHB Research Center (1993) discusses two approaches to the estimation of uncertainties in the Measured Performance Rating System or MPR. One compared measured fuel consumption to MPR predictions for four homes; the other used computer simulations. The billing data related well to outdoor temperatures in two cases, but in the other two they did not. An additional effort looked at measurement bias and scatter, and how they propagate into the estimates of building load coefficient and heating system efficiency $\left(\eta_{\text {system }}\right)$.

What is relevant to the present discussion is the authors' statement that their analysis indicates that the MPR method can achieve measurement uncertainties in the heating load coefficient of 1 to 5 percent at the 95\% confidence level. For comparison with 
the results reported in the other papers discussed here, this would correspond to a standard deviation of under $3 \%$.

The authors discussed greater deviations of the MPR results from "actual" values, where both were simulated via a computer model. However, they say with regard to these simulations that "we cannot be sure of the models."[NAHB 1993, page D-46] In the absence of more information, we do not think that the computer simulation results contained in this paper shed much light, one way or the other, on the acceptability of coheating in duct efficiency testing, but they are mentioned here for completeness.

Saunders et al. 1994 reported on seven heating system retrofits that included actions on both heating equipment and ducts. The MPR method was used to assess system efficiency. In one house, five separate tests were performed. The values obtained for the heating load coefficient, unadjusted for changes in infiltration, showed a standard deviation of $4 \%$ of the mean value. After adjustment for infiltration, this standard deviation increased to $5.5 \%$ of the mean. That is somewhat surprising, since one would expect the variance to decrease after infiltration corrections, but perhaps the "raw" variance was abnormally low due to chance.

In coheating tests done on two houses in New York state, Andrews et al. (1995b) obtained heating load coefficients of 456 , 421,431 , and $463 \mathrm{Btu} / \mathrm{F}-\mathrm{h}$ in one house and 333 and $366 \mathrm{Btu} / \mathrm{F}-\mathrm{h}$ in the other. (The first house was the same one tested by Jones et al. 15 years previously. The difference of $110 \mathrm{Btu} / \mathrm{F}-\mathrm{h}$ was explained by measured differences in air infiltration.) These values lead to standard deviations of $3.9 \%$ of the mean value for the first house and $4.7 \%$ of the mean value 350 for the second house. The average indoor-outdoor temperature difference was $32 \mathrm{~F}$ for the first house and $38 \mathrm{~F}$ for the second house.

The findings discussed above are summarized in Table 2 .

Although the data are not as voluminous as one might wish, they do give a fairly consistent picture of the achievable level of repeatability for coheating measurements taken close together in time. They appear to support a reasonable expectation to achieve a variability in the HLC, measured in terms of one standard deviation, of 3 to 5 percent, especially if the measurements are done for reasonably large indoor-outdoor temperature differences (30 F or greater). With care, the lower half of this range should be achievable, especially as the method becomes more widely practiced. If it is desired to reduce the random error to less than $3 \%$, significant refinements in the method will likely be required. 
Table 2. Random Errors in Heating Load Coefficients Measured Using Coheating.

\begin{tabular}{|c|c|c|c|}
\hline $\begin{array}{l}\text { Citation (First } \\
\text { Author and Date) }\end{array}$ & $\begin{array}{l}\text { Random } \\
\text { Error }^{1} \\
\end{array}$ & $\begin{array}{l}\text { No. of } \\
\text { Values }\end{array}$ & Conditions \\
\hline \multirow[t]{2}{*}{ Sonderegger 1979} & $9 \%$ & 8 & All values \\
\hline & $3.3 \div$ & $\begin{array}{c}4 \\
\text { pairs }\end{array}$ & $\begin{array}{l}\text { Pairs of values taken close } \\
\text { in time for same purpose }\end{array}$ \\
\hline Duffy 1989 & $3.3 \%$ & -- & $\begin{array}{l}\text { Engineering judgment, } 30 \mathrm{~F} \\
\text { indoor-outdoor } \Delta \mathrm{T}\end{array}$ \\
\hline Jones 1984 & $1.3 \%$ & 2 & $\begin{array}{l}\text { Two values } 20 \text { days apart, } \\
\text { Long Island, New York }\end{array}$ \\
\hline \multirow[t]{2}{*}{ Subbarao 1990} & $16.8 \%$ & 13 & $\begin{array}{l}\text { "Raw" values for tests } \\
\text { conducted over } 5 \text { months. }\end{array}$ \\
\hline & $2.8 \%$ & 13 & $\begin{array}{l}\text { "Corrected" values accoun- } \\
\text { ting for weather variables } \\
\text { other than temperature }\end{array}$ \\
\hline \multirow[t]{2}{*}{ Saunders 1984} & $4 \%$ & 7 & "Raw" values \\
\hline & $5.5 \%$ & 7 & Corrected for infiltration \\
\hline \multirow[t]{2}{*}{ Andrews $1995 \mathrm{~b}$} & $3.9 \%$ & 4 & $\begin{array}{l}\text { Tests on a house in Iong } \\
\text { Island, New York }{ }^{2}\end{array}$ \\
\hline & $4.7 \%$ & 2 & $\begin{array}{l}\text { Tests on a house in } \\
\text { Watertown, New York }\end{array}$ \\
\hline
\end{tabular}

Note 1: One standard deviation as percent of mean value.

${ }^{2}$ Note 2: Same house as that used in Jones et al. 1984

Systematic Error. A recent paper [Iiu and Claridge 1995] details three ways that a coheating test can underestimate the true UA value of a building. They simulated a $1600 \mathrm{ft}^{2}$ house and then tested their theory in a test cell constructed for the purpose. The factors that can throw off a coheat test were given by the authors as:

- Impact of neglecting solar radiation through windows.

- Impact of neglecting solar radiation through walls and roof.

- Impact of neglecting relatively higher daytime temperature.

With respect to solar radiation through windows, this reduced the heating load on the model house by an average of $700 \mathrm{~W}$ between midnight and $7 \mathrm{a.m.}$, and by an average of approximately $1300 \mathrm{~W}$ between $6 \mathrm{p} . \mathrm{m}$. and midnight. The authors say that covering the windows with an "opaque" material is a good idea. We would agree, but would substitute the word "reflective" for "opaque." 
Solar radiation through opaque walls and roof cannot be so easily disposed of. The authors project a reduction in heating load by an average $85 \mathrm{~W}$ from midnight to $7 \mathrm{a.m}$. and by an average of approximately $200 \mathrm{~W}$ between $6 \mathrm{p} . \mathrm{m}$. and midnight. This would reduce the coheat energy required to maintain indoor temperature and thus yield a HLC that is lower than the true UA value. The relevance to the coheating determination of system efficiency is that the heating system tests should experience the same reduction in load, and therefore this effect should not have any impact on the measured system efficiency. This would be true as long as each test experiences the same conditions, $i . e$. the same number of hours before midnight as after and the same incident sunlight before equivalent tests.

One possible source of difficulty could occur in a protocol where the heating system was tested throughout one night and coheating was done on the second night. Then if (say) the system test followed a sunny day and the coheat test followed a cloudy day, the HSC would be (relatively) too low in relation to the HLC, and the system efficiency (ratio of HLC to HSC) would be artificially high.

Even the "flip-flop protocol" currently envisioned for Standard 152, in which both tests are performed on both nights but with the order reversed, would not eliminate this effect, although it would reduce it. That is because the effect of a difference in solar insolation would be more profound on the before-midnight tests than on the after-midnight ones. That is to be expected because by midnight the effect of solar gain has largely (though not completely) dissipated. In other words, whichever system (heating system or coheaters) had its before-midnight test following the sunny day would use less energy relative to the other system than would be the case if they both faced similar conditions lingering from the day before. This would bias the system efficiency upward or downward. This effect is quantified in the next section.

The third source of bias, the impact of neglecting relatively higher daytime temperature, refers to the fact that the heating load caused by temperature difference has a time delay with respect to that temperature difference. This can cause the calculated HLC, which uses current temperature differences, to deviate from the true UA value because the heating load is in part caused by temperature differences that occurred some time in the past. Liu and Claridge quote possible deviations of up to $13 \%$ for an outdoor temperature of $-7{ }^{\circ} \mathrm{C}$ and as much as $21 \%$ for an outdoor temperature of $5^{\circ} \mathrm{C}$.

A quantitative discussion of this paper's findings in the context of duct system efficiency measurements is given in the next section. 
Systematic Error Quantified in Duct Efficiency Testing. The paper of Liu and Claridge raises questions about the ability of coheating to measure accurately the UA value of a house. This is, however, a different question from whether it is possible to obtain accurate relative values of energy use by comparing an in-situ heating system with coheaters for equivalent indoor temperatures and equivalent (or corrected-for) outdoor conditions.

Let us look at the three sources of error considered by Liu and Claridge. The first of these is solar gain through windows. The coheating test method currently envisioned for ASHRAE Standard 152 prescribes covering the windows on the east, south, and west sides of the house with a reflective material before beginning the test. In view of the potentially large impact of the solar gain, it would probably be well to prescribe that the windows be covered well in advance of testing, possibly for the entire day preceding the first test night.

To quantify the magnitude of Liu and Claridge's second source of error--solar radiation through opaque walls and roof--we performed the following simplified calculation. Assume a worst case where one day is sunny and the other cloudy. For the case where the coheaters are tested before midnight following the sunny day and after midnight following the cloudy day (with the heating system tested in the alternate periods), the coheat system will experience a net reduction in load of $200 \mathrm{~W} \times 6 \mathrm{~h}$ or $1.2 \mathrm{kWh}$ (relative to the heating system) in the before-midnight tests, while the heating system will have a net reduction in load (relative to the coheaters) of $85 \mathrm{~W} \mathrm{X} 6 \mathrm{~h}$ or $0.5 \mathrm{kWh}$ in the aftermidnight tests, so, net, the coheaters have a $0.7 \mathrm{kWh}$ advantage.

The same model gives an actual average load due to temperature differences, during the coheat period, of $2000 \mathrm{~W}$ when the outdoor temperature is $5^{\circ} \mathrm{C}$ and $3800 \mathrm{~W}$ when the outdoor temperature is -7 ${ }^{\circ} \mathrm{C}$ (implying a balance point of $18^{\circ} \mathrm{C}$ ). The coheaters then should use $24 \mathrm{kWh}$ of energy over 12 hours under the warmer conditions and $46 \mathrm{kWh}$ under the colder conditions. The percentage error associated with the above effect would be $(0.7 / 24) \times 100=3 \%$ under the warmer conditions and $(0.7 / 46) \times 100=1.5 \%$ under the colder conditions. This would not appear fatal, especially when it is considered to be the maximum that occurs when one day is very sunny and the other is very cloudy. Nevertheless, it cannot be ignored.

One should note that if all the system testing was done on one night and all the coheat testing on the other, then the bias due to opaque-surface solar gains could be as high as $7 \%$ if one day was warm and sunny and the other day was cloudy. This is an argument in favor of the flip-flop protocol.

To estimate the impact of Liu and Claridge's third source of error--neglecting relatively higher daytime temperature--we used 
their values for the percentage deviations between midnight and 7 a.m., together with reading their graph to get similar numbers for 6 p.m. to midnight. In one case it appears they may have misread their own graph. somewhat; in that case the best value from the graph was used. These results are shown in Table 3.

In this example, it is assumed that the first system test is performed from $6 \mathrm{p} . \mathrm{m}$. to midnight on the colder day, followed by coheating in the early morning hours. A warming trend then sets in, so that on the following night the temperature profile is that appropriate to a day with an average temperature of $5{ }^{\circ} \mathrm{C}$. Then the coheating test is done before midnight and the heating system test after. We can compute the average percentage bias by noting the actual heating loads given by the authors' model for the six hours before and after midnight (shown in the last two rows of the table).

Table 3. Summary of Biases Reported in Liu and Claridge 1995, Arising from Phase Lag of Heating Load with Respect to Temperature. Numbers Referring to Coheating Tests are Highlighted.

\begin{tabular}{||l|c|c||}
\hline \multicolumn{1}{|c|}{$\begin{array}{c}\text { Average Outdoor } \\
\left.\text { Temperature ( }{ }^{\circ} \mathrm{C}\right)\end{array}$} & -7 & 5 \\
Parameter & & $+7 \%$ to $-20 \%$ \\
\hline $\begin{array}{l}\text { Range of Percentage Errors, } \\
\text { Before-Midnight Tests }\end{array}$ & $+5 \%$ to $-13 \%$ & $-20 \%$ to 0 \\
\hline $\begin{array}{l}\text { Range of Percentage Errors, } \\
\text { After-Midnight Tests }\end{array}$ & $-13 \%$ to 0 & $-6.5 \%$ \\
\hline $\begin{array}{l}\text { Average Percentage Bias, } \\
\text { Before-Midnight Tests }\end{array}$ & $-4 \%$ & $-10 \%$ \\
\hline $\begin{array}{l}\text { Average Percentage Bias, } \\
\text { After-Midnight Tests }\end{array}$ & $-6.5 \%$ & 10 \\
\hline $\begin{array}{l}\text { Heating Ioad, 6 Hours } \\
\text { Before Midnight (kW) }\end{array}$ & 21 & 14 \\
\hline $\begin{array}{l}\text { Heating Load, 6 Hours } \\
\text { After Midnight (kW) }\end{array}$ & 25 & \\
\hline
\end{tabular}

The bias for the coheating tests is then:

$$
\frac{25(-0.065)+10(-0.065)}{25+10}=-0.065
$$

or 6.5\%. Similarly, the bias for the heating system tests is:

$$
\frac{21(-0.04)+14(-0.10)}{21+14}=-0.064
$$


or $6.4 \%$. Hence the bias for the coheat tests and that for the system tests is nearly the same, which means that the system efficiency should be virtually unaffected. It must be admitted that if the same model were applied to a different house, the results might not be this close. Also, if the authors' quotation of the range of values for post-midnight bias at $5 \mathrm{C}$ is reversed with that for the pre-midnight bias (to agree with their text and not with their graph), this would result in a $2.5 \%$ difference between the bias in the coheat tests and in the system tests.

Also to be noted is that if the coheat tests are all done on one night and the system tests all on the other, then a $-5.4 \%$ bias results on the colder night and a $-8.5 \%$ bias on the warmer one, meaning that the system efficiency would be biased up or down by approximately 3\% depending on which test was done on the colder night. As in the case of solar gains on opaque surfaces, this favors the flip-flop protocol.

The result of these analyses is to conclude that, although coheating may give rise to significant biases if the objective is to obtain an absolute value of the steady-state heat-loss coefficient (UA) for a house, we are in much better shape if the objective is only to obtain relative values for one heating system vs. another. This is especially true for the flip-flop protocol.

Related to these issues are some considerations on testing recently done by the author of this paper (with coworkers). [Andrews et al. 1995] Among the issues discussed in this paper is the question of whether the efficiency of attic ductwork might be affected by solar gains on the day before the testing is done.

A house with some ductwork in the basement and some in the attic was tested using the flip-flop protocol. Four nights of testing (two complete flip-flop protocols) were performed. The system efficiency values obtained for this house were:

$\begin{array}{llll}\text { Test Dates } & \begin{array}{l}\eta_{\text {system }} \\ \text { Ducts }\end{array} \text { First } & \begin{array}{l}\eta_{\text {system }} \\ \text { Coheat First }\end{array} & \begin{array}{l}\eta_{\text {system }} \\ \text { Average }\end{array} \\ 3 / 15-16 \& 16-17 & 0.686 & 0.578 & 0.632 \\ 3 / 17-18 \& 20-21 & 0.682 & 0.506 & 0.594\end{array}$

Although these average values were fairly close, there is considerable variation in the individual system efficiencies. Some of this is probably due to thermal mass effects. Thermal mass was intentionally added to the house as storage for its passive solar features. Thermal mass retards the response of a building to changes in the indoor-outdoor temperature difference. We concluded that, in particular, the especially low value of 0.506 obtained on March 20-21 is probably due in part to thermal mass effects, in line with the fact that there was a significant temperature rise after midnight on that night. 
However, thermal mass cannot explain all of the variation. For example, for the pair of tests on 3/15-16 and 3/16-17, the system efficiency was higher when the ducts went first even though the outdoor temperature profile was relatively flat during those two nights. An alternative explanation (suggested by Mark Modera, Lawrence Berkeley Laboratory) could be that the portion of the duct system in the attic benefitted from residual solar gain when the heating system ran first (before midnight) but that little or no solar-derived heat was left in the attic after midnight. (Note that this is natural solar gain; there is no active or passive solar system on the roof.) In that case we would expect the HSC's to be lower when the ducts were tested first, which was in fact observed.

The problem here is that, if the difference is due to solar gain in the attic, its impact on testing attic ducts goes beyond the general effect noted by Liu and Claridge. They considered whole-house solar gain on opaque surfaces, and this would be the same whether the heating system or the coheaters are running. Here, however, we are focusing on solar gain received by the attic, which could affect the ducts in the attic but not the coheating system.

Because the coheating system would not be affected very much by attic temperature (in view of the fact that the second-floor ceiling is well insulated), the HLC's should not be biased on the basis of which system was tested first. This is consistent with our data: the highest and the lowest of our four HLC values were obtained when the coheating system was tested first.

The flip-flop protocol attempts to compensate for this and other artifacts of the order of testing by reversing the order during the second test. However, there is a concern that, for ducts in an attic, the measured value of the HSC when the ducts are tested first might depend significantly on whether the day before the test was sunny or not, while the HLC for the coheating tests would not depend very much on this variable. That would mean that the measured system efficiency is dependent on solar gain, despite the attempt to eliminate this variable by covering the windows with reflective foil. In other words, in the case of attic ductwork, variation in HSC with the time of the test may not be an artifact of the test method at all, but may be a real effect. Attic ducts used for heating may be more efficient during and shortly after sunny periods. (The reverse would then be true for cooling.) In that case, this will have to be recognized in any test method, not just the one involving coheating. In the end, it may be that, for attic ductwork, standard 152 will need either to account for the dependence of system efficiency on solar gain or to minimize it by restricting the ambient conditions under which systems with ducts in an attic can be tested. 
Similar measurements on a low-mass house with ducts in a vented crawl space gave much closer agreement between the two nights of a coheating test. The values were 0.507 and 0.532 . This suggests that coheating may be easier to apply in some types of houses than others, or at least may be applied in a broader range of weather conditions.

To. sum up the above discussion, coheating appears to be able to give good results relative to other tests done at nearby times, even if there may be an absolute bias. For certain types of houses, specifically those with attic ducts and/or very large thermal mass, it may be necessary to introduce correction factors or else to restrict the range of ambient conditions under which coheating tests leading to duct efficiency are accepted. This problem, if real, will not be restricted to coheating tests, but is very probably a more general issue characteristic of testing attic duct systems. In that case, any test method will need to address it.

Consistency of Coheating Results with Physical Constraints. Another way of assessing the accuracy of coheating as applied to thermal distribution efficiency is to look at the range of results obtained with it and see whether they are consistent with the laws of physics and with reasonable assumptions concerning equipment and duct systems.

For example, Saunders et al. 1994 reported on seven heating system retrofits that included actions on both heating equipment and ducts. The MPR method was used to assess system efficiency. System efficiencies for the 7 houses ranged from 23\% to $41 \%$ before retrofitting. The house with $23 \%$ efficiency then had a new furnace installed, and its ducts were sealed and insulated; after this was done the heating system efficiency rose to $80 \%$. Not only was this dramatic improvement, it was to a reasonable level in terms of expectations. That is, had the post-retrofit system efficiency risen to more than $100 \%$ (or indeed to much above $80 \%$ given the fact that no furnace is $100 \%$-efficient and current duct sealing methods have their limitations), this would have indicated a deficiency in the method. Had it not risen to within a reasonable fraction of $100 \%$ despite the extensive measures taken to improve the furnace and duct efficiency, again the test method would have been suspect.

Perhaps the best evidence to date for the accuracy of coheating measurements of system efficiency is contained in olson et al. 1993. Two of the 24 homes tested had ducts in the conditioned space. These two homes had measured system efficiencies of $99 \%$ and $97 \%$, in contrast to the 22 with ducts not in the conditioned space, which averaged $71 \%$ system efficiency. With ducts in the conditioned space and an electric furnace, one expects a system efficiency very close to $100 \%$. (It might be slightly less than $100 \%$ if the duct. system, though in the conditioned space, nevertheless pressurizes zones unequally, or if, 
despite the builder's best efforts, there are undetected flow paths from the ducts to the outside.) The only negative thing one can say about these results is that there aren't enough houses to make a firm conclusion. This is one case where the researcher's common conclusion that "more research is needed" happens to be true. Nevertheless, the results to date appear very promising.

What About Cooling?

Although testing in the cooling mode is beyond the scope of this report, a few words may be in order. One obvious drawback of electric coheating as a test method is that there is no electric co-cooling. The question therefore must be asked: is there any reasonable way to circumvent this difficulty?

Two approaches have been suggested. One way could be to use a cooling loop that employs chilled water or the heat-absorbing capacity of condensed refrigerant to remove heat from the conditioned space, with the rejection of this heat carried out in equipment located outside the building. Reliable means for measuring the heat removed (sensible and latent) would have to be provided. Some work on this idea is discussed in Andrews (1993).

Another possible approach would be to use coheaters to inject a known incremental cooling load to the building. This option is discussed in sonderegger et al. (1979), and in fact some tests of this nature were carried out. The method does not seem to have been explored to any significant degree since then, however. 